\title{
Solidariedade InTERgeracional de Avós com Netos com DeficiênCia: Análise Bibliométrica e de ConTeúdo ${ }^{1}$
}

\author{
INTERGENERATIONAL SOLIDARITY OF GRANDPARENTS WITH DISABLED \\ GRANDCHILDREN: BIBLIOMETRIC AND CONTENT ANALYSIS
}

\author{
Maria Cristina Piumbato Innocentini HAYASHI ${ }^{2}$ \\ Carlos Roberto Massao HAYASHI ${ }^{3}$ \\ Carolina REBELLATO 4 \\ Claudia Maria Simóes MARTINEZ 5
}

\begin{abstract}
RESUMO: Os avós, sobretudo as avós, são fontes importantes de apoio para famílias de crianças com deficiência. A presença dessas avós no contexto da vida diária propicia trocas de apoio que podem funcionar como mecanismos de proteçâo e promover a resiliência familiar. Nesse contexto, a Solidariedade Intergeracional (SI) colabora para o enfretamento de estressores e consequentes impactos na saúde dos indivíduos. Nesse sentido, este artigo objetiva compreender como se configura a produção científica da literatura científica nacional e internacional que aborda a SI entre avós e mães de crianças com deficiência. A pesquisa bibliográfica, exploratória-descritiva foi realizada por meio de análise bibliométrica e de conteúdo. A coleta de dados ocorreu no Portal de Periódicos Capes, mediante a utilização de expressôes de busca extraídas da literatura. Após os critérios de inclusão e exclusão, foi constituído o corpus de análise $(\mathrm{n}=18)$. Os resultados informam maioria feminina nas produçóes em coautorias, no período de 1994 a 2016. Há 16 títulos editados em cinco países. Na maioria dos estudos, foram identificadas as dimensóes funcional e afetiva. A partir dos desfechos dos estudos, são discutidos os seguintes subtemas: avós como fontes de apoio; atuação dos profissionais com a família, incluindo os avós; níveis de estresse e conflitos; diferenças e semelhanças entre avós de netos com ou sem deficiências; e dados culturais e universais. A presente pesquisa reforça a complexidade das relaçóes familiares e a multidimensionalidade da SI, que é construída ao longo do tempo e atravessada pelo contexto social e cultural.
\end{abstract}

PALAVRAS-CHAVE: Solidariedade Intergeracional. Família. Criança com deficiência. Produção científica. Análise bibliométrica.

\begin{abstract}
Grandparents, especially the grandmothers, are important sources of support for families of children with disabilities. The presence of these grandmothers in the context of the daily life of these families provides intergenerational support exchanges that can act as protection mechanisms and promote the family resilience. In this context, intergenerational solidarity (IS) contributes to facing stressors and consequent impacts on the health of individuals. In this sense, this article aims to understand how the scientific production of the national and international scientific literature that addresses IS among grandparents and mothers of children with disabilities is configured. The bibliographic and exploratory-descriptive research was performed through bibliometric and content analysis. Data collection took place in the Portal of Capes journals, using search expressions extracted from the literature. After the inclusion and exclusion criteria, the corpus of analysis was created $(\mathrm{n}=18)$. The results reported female majority in co-authored productions, from 1994 to 2016. There are 16 titles published in five countries. In most of the studies, the functional and affective dimensions were identified. From the study outcomes, the following subtopics are discussed: grandparents as sources of support; performance of professionals with the family, including grandparents; stress and conflict levels; differences and similarities between grandchildren with or without disabilities; and cultural and universal data. The present research reinforces the complexity of family relationships and the multidimensionality of intergenerational solidarity, which is built over time and traversed by the social and cultural context.
\end{abstract}

KEYWORDS: Intergenerational solidarity. Family. Disabled child. Scientific production. Bibliometric analysis.

\footnotetext{
${ }^{1}$ https://doi.org/10.1590/1980-54702021v27e0025

${ }^{2}$ Doutorado em Educação. Professora Titular no Departamento de Ciência da Informação e nos Programas de Pós-Graduação em Ciência, Tecnologia e Sociedade (PPGCTS), Educação (PPGE) e Educação Especial (PPGEEs). Universidade Federal de São Carlos (UFSCar). São Carlos/São Paulo/Brasil. E-mail: dmch@ufscar.br. ORCID: https://orcid.org/0000-0003-1250-3767

${ }^{3}$ Doutorado em Educação. Professor Associado do Departamento de Ciência da Informação e nos Programas de Pós-Graduação em Educação (PPGE) e Ciência, Tecnologia e Sociedade (PPGCTS). Universidade Federal de São Carlos (UFSCar). São Carlos/ São Paulo/Brasil. E-mail: massao@ufscar.br. ORCID: http://orcid.org/0000-0003-1481-5545

${ }^{4}$ Doutorado em Educação Especial. Professora Adjunta do Departamento de Terapia Ocupacional. Universidade Federal do Rio de Janeiro (UFRJ). Rio de Janeiro/Brasil. E-mail: crebellato.to@medicina.ufrj.br. ORCID: https://orcid.org/0000-0001-8359-6724

${ }^{5}$ Doutorado em Educação. Professora Titular do Departamento de Terapia Ocupacional e nos Programas de Pós-Graduação em Terapia Ocupacional e Educação Especial (PPGEEs). Universidade Federal de São Carlos (UFSCar). São Carlos/São Paulo/Brasil. E-mail: claudia@ufscar.br. ORCID: https://orcid.org/0000-0002-8997-2767
} 


\section{INTRODUÇÁo}

Solidariedade tem sido um constructo utilizado em diferentes campos do conhecimento. Interessa-nos estudar a Solidariedade Intergeracional Familiar (SIF) nas situaçóes em que há uma criança com deficiência em seu núcleo. $\mathrm{O}$ foco da presente pesquisa recai, assim, no campo de estudos sobre o desenvolvimento humano, sendo destacados os conceitos de vulnerabilidade social e Solidariedade Intergeracional (SI).

Diferentes campos do saber e práticas utilizam o conceito de vulnerabilidade que, de uma forma geral, está relacionada a situaçôes de insegurança e risco em diferentes dimensóes da vida (Luvizaro \& Galheigo, 2011). Para pesquisadores da Organização das Naçóes Unidas para a Educação, a Ciência e a Cultura (UNESCO) e Banco Interamericano de Desenvolvimento (BID), a vulnerabilidade social é compreendida como

o resultado negativo da relação entre a disponibilidade dos recursos materiais ou simbólicos dos atores, sejam eles indivíduos ou grupos, e o acesso à estrutura de oportunidades sociais, econômicas, culturais que provêm do Estado, do mercado e da sociedade. Esse resultado se traduz em debilidades ou desvantagens para o desempenho e mobilidade social. (Abramovay et al., 2002, p. 29)

Nesse enfoque, a vulnerabilidade social está atrelada às condiçóes sociodemográficas e do suporte social que esse grupo possui, associado aos direitos sociais que acessam e usufruem (Abramovay et al., 2002); extrapola, portanto, o fenômeno de pobreza e da falta de acesso a bens e serviços, considera também a fragilidades de vínculos afetivo-relacionais (Carmo \& Guizardi, 2018).

No contexto da infância, a família exerce um papel fundamental no desenvolvimento da criança, por meio dos relacionamentos estabelecidos no cotidiano, em uma perspectiva bidirecional e sistêmica, em que há influências mútuas entre seus membros (Milanez et al., 2019). A perspectiva de protagonismo da família nuclear na função de cuidado e proteção de seus membros pode ficar comprometida frente à presença de uma criança com deficiência, por diversos motivos: aceitação da deficiência, instabilidade emocional, sensação de confiança, mudança de papéis, acesso a serviços, vivência prática de cuidado da criança em diferentes ambientes e conflito relacional (Barbosa et al., 2012). Esse fato pode requerer a aproximação e a ampliação de práticas de cuidado envolvendo outras geraçóes familiares para apoio emocional e funcional de pais de crianças com alguma deficiência (Yamashiro \& Matsukura, 2014).

As pesquisas sobre as relaçóes familiares e intergeracionalidade estão fortemente embasadas na teoria de SI proposto por Bengston e Roberts (1991). A teoria envolve uma taxonomia e modelo multidimensional sólido e duradouro que possibilita compreender os padróes de coesão intergeracional entre pais e seus respectivos filhos adultos (Bengtson \& Roberts, 1991; Leme et al., 2016). Os referidos autores descreveram inicialmente seis dimensóes como componentes essenciais da SI: Associativa, Afetiva, Consensual, Funcional, Normativa, Estrutural. Após críticas ao modelo quanto à supervalorização em abordar aspectos harmoniosos e positivos das relaçóes familiares (Hogerbrugge \& Komter 2012), foi incorporada a dimensão conflito, que se refere aos aspectos complexos, mas naturais e próprias dos relacionamentos, como as tensôes e as divergências presentes nas interaçôes familiares (Clarke et al., 1999; Bengtson \& Martin, 2001; Bengtson et al., 2002). Todas as dimensôes estão estruturalmente relacionadas 
entre si; desse modo, níveis de solidariedade em uma dimensão afeta níveis em outras dimensôes ao longo do ciclo vital (Hogerbrugge \& Komter, 2012; Leme et al., 2016).

O processo de transmissão de conhecimento, cultura e prática de cuidado por meio da interdependência e interação geracional sofre influência de fatores pessoais, familiares e sociais de ambas as geraçóes (Bengston \& Roberts, 1991; Leme et al., 2016). Apesar do referencial teórico de SIF enfocar a díade pai-filhos adultos, atualmente é possível expandir a compreensão para relaçóes intergeracionais entre netos e avós, ao considerar-se, sobretudo, a complexidade e multidimensionalidade das interaçóes familiares e a longevidade (Leme et al., 2016).

No Brasil, o número de idosos na população global aumentará significativamente até o ano de 2050 (Miranda et al., 2016). Essa transição demográfica associada ao aumento da expectativa de vida oferta um novo cenário de transferências intergeracionais a ser explorado (Centro Internacional de Longevidade Brasil [ILC-BR], 2015). Na prática, observa-se um aumento no número de idosos que provêm recursos para seus filhos adultos e netos - nesse contexto, é importante considerar também as diferenças de gênero, uma vez que esse apoio pode se concentrar mais entre mulheres de diferentes geraçóes (ILC-BR, 2015). Nesse âmbito, estudos têm se dedicado a investigar as relaçóes entre avós e pais de crianças com deficiência e indicado que a presença de um neto com deficiência traz influência para o estabelecimento das relações familiares e constituição dos papéis de avós (Mirfin-Veitch et al., 1997; Woodbrigde et al., 2011), impactando diretamente os componentes da SI.

Diante da diversidade de arranjos familiares e das distintas condições das famílias para criarem as crianças, entre elas as com deficiência, hipotetiza-se que, na rede de relacionamento familiar, as avós se constituem fontes de suporte às suas filhas no processo de cuidar e de educar uma criança com restrição de participação e limitaçóes na execução de atividades cotidianas. $\mathrm{O}$ presente estudo foi norteado pela busca de respostas para a seguinte questão de pesquisa: Como se configura a produção científica sobre a temática da Solidariedade Intergeracional familiar entre avós e mães? Para responder a essa questão, realizou-se uma pesquisa com os seguintes objetivos: a) compreender como a literatura científica nacional e internacional aborda a SI de avós de crianças com deficiência; b) selecionar e analisar artigos científicos originais que tratam dessa temática publicados em periódicos nacionais e internacionais; c) realizar análise quantitativa e qualitativa dessa produção científica.

\section{Método}

A pesquisa realizada é de tipo bibliográfica, de nível exploratório-descritiva que visa à compreensão quantitativa e qualitativa da produção científica selecionada para análise; também pode ser considerada como um estudo do tipo "estado do conhecimento" (Hayashi \& Gonçalves, 2018). Em termos metodológicos as abordagens da análise bibliométrica e da análise de conteúdo foram combinadas. A primeira visa extrair informaçóes úteis para a compreensão da estrutura social e intelectual de um campo científico mediante a análise dos padróes de comunicação científica presentes nos documentos analisados (Silva et al., 2011). A segunda refere-se a um conjunto de técnicas de análise que possibilita a descrição objetiva, sistemática e quantitativa que permite a inferência de conhecimentos extraídos dos textos (Bardin, 2011). 
A fonte de dados escolhida foi o Portal de Periódicos da Coordenação de Aperfeiçoamento de Pessoal de Nível Superior (CAPES), por reunir, em um único espaço virtu$\mathrm{al}^{6}$, publicaçóes científicas. $\mathrm{O}$ acervo é constituído por 37.818 periódicos, além de várias bases, resultando na disponibilidade de mais de 266.272 documentos eletrônicos, dentre capítulos de livros, relatórios, anais, manuais, guias e outros. A coleta de dados ocorreu em janeiro de $2019 \mathrm{e}$ os artigos selecionados para análise foram publicados entre 1994 e 2016, mediante a utilização do mecanismo busca simples utilizando as seguintes expressóes no idioma inglês, considerando que ela é a língua franca da ciência: "intergenerational solidarity" disabilities; "grandparents intergenerational solidarity"; "grandchildren intergenerational solidarity" "grandmothers intergenerational solidarity", o que resultou em 168 artigos. Após a aplicação dos critérios de inclusão - artigos originais, sem limitação temporal e de idiomas visando incluir o maior número de registros - e de critérios de exclusão - artigos sem texto completo e não adequados ao escopo da pesquisa, embora apresentassem as expressóes de busca no título e no resumo, mas que não estavam integralmente representadas no conteúdo dos artigos - obteve-se o corpus de análise $(\mathrm{n}=18)$ (Figura 1).

\section{Figura 1}

Fluxograma do processo de seleção do corpus da pesquisa

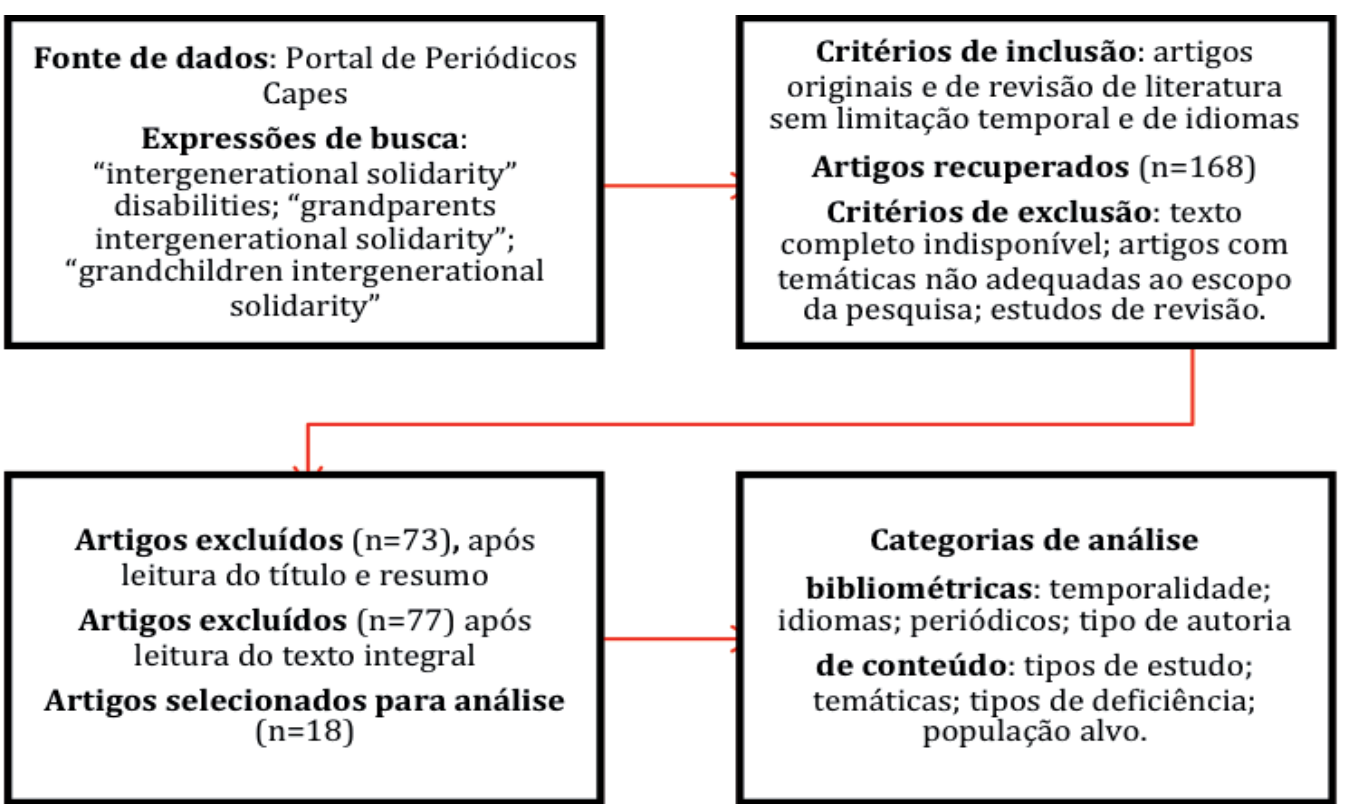

Em seguida, os dados coletados foram inseridos em uma planilha Excel para registro e elaboração de categorias quantitativas (bibliométricas) e qualitativas (de conteúdo), representadas pelas seguintes variáveis: a) perfil dos autores, autorias e publicaçóes: tipo de autoria (individual ou coautoria); evolução temporal, título, países e idioma das publicaçóes; b) conteúdo dos artigos: tipos de deficiências e população-alvo dos estudos; países ou regióes focalizadas; temáticas e dimensóes da SI abordadas nos estudos. Esses dados foram tratados mediante esta-

${ }^{6}$ Ver http://www.periodicos.capes.gov.br/index.php?option=com_pinstitucional\&Itemid=104. 
tística descritiva visando à produção e à análise de indicadores bibliométricos. Quanto às categorias da análise de conteúdo, após a recuperação dos artigos por duas pesquisadoras, foi realizada a leitura integral, seguindo as etapas de pré-análise, exploração e interpretação dos textos. Para assegurar a fidedignidade dos dados qualitativos, ambas realizaram de forma independente uma proposta de categorização dos temas relativos aos desfechos dos estudos. No processo de validação das categorias encontradas, houve a fusão de algumas temáticas, após apreciação por um terceiro pesquisador, o que possibilitou o enquadramento dos temas em cinco categorias.

\section{Resultados E Discussão}

Nesta seção, abordam-se a temporalidade dos artigos, o perfil dos autores e das autorias, os países focalizados, tipos de deficiência e população-alvo dos estudos, as Dimensôes da Solidariedade Intergeracional (SI) e, por fim, os objetivos e os desfechos dos estudos.

\subsection{Temporalidade dos aRTigos}

A Figura 2 apresenta a distribuição anual dos artigos no período entre 1994 e 2016.

\section{Figura 2}

\section{Distribuição anual dos artigos}

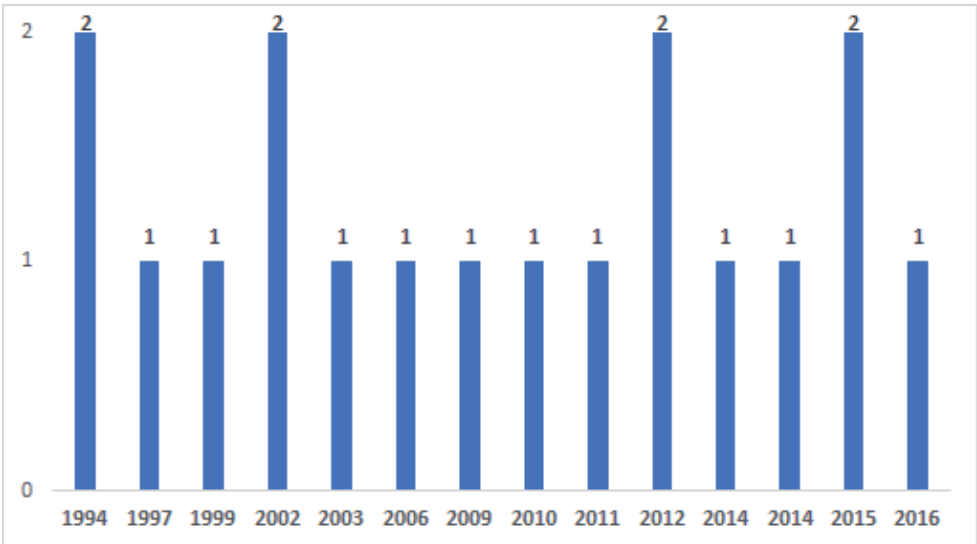

Observou-se um padrão constante na distribuição anual dos artigos, mas que revela a existência de poucos estudos publicados efetivamente em quinze anos de publicação, haja vista as lacunas nos anos de 1995-1996, 1998, 2000-2001, 2004-2005, 2007-2008 e 2013. Esses dados sugerem que a temática da SI em famílias com crianças com deficiência ainda é pouco explorada pela literatura científica, com muito ainda a ser pesquisado.

A investigação sobre os periódicos que publicaram os artigos (Tabela 1) revelou a existência de diferentes títulos $(n=16)$ editados em diferentes países $(n=5)$. 


\section{Tabela 1}

Distribuição dos artigos por títulos de periódicos e países

\begin{tabular}{l|l|c}
\multicolumn{1}{c|}{ Título } & \multicolumn{1}{c}{ País } & Artigos \\
\hline Education \& Treatment of Children & EUA & 2 \\
\hline Journal of Applied Research in Intellectual Disabilities & Inglaterra & 2 \\
\hline Academic Journal of Business, Administration, Law and Social Sciences & Áustria & 1 \\
\hline Australasian Journal on Ageing & Austrália & 1 \\
\hline Child: Care, Health \& Development & Inglaterra & 1 \\
\hline Estudos Interdisciplinares sobre o Envelhecimento & Brasil & 1 \\
\hline Family Relations & Inglaterra & 1 \\
\hline International Journal of Developmental Disabilities & Inglaterra & 1 \\
\hline Issues in Comprehensive Pediatric Nursing & EUA & 1 \\
\hline Journal of Advanced Nursing & Inglaterra & 1 \\
\hline Journal of Aging Studies & EUA & 1 \\
\hline Journal of Child and Family Studies & EUA & 1 \\
\hline Journal of Early Intervention & EUA & 1 \\
\hline Psicologia em Estudo & Brasil & 1 \\
\hline Psicologia: Teoria e Pesquisa & Brasil & 1 \\
\hline Revista Brasileira de Educação Especial & Brasil & 1 \\
\hline Total & & $\mathbf{1 8}$ \\
\hline
\end{tabular}

Os artigos sobre SI foram publicados majoritariamente em periódicos na Inglaterra $(n=5)$, seguido pelos Estados Unidos $(n=4)$ e pelo Brasil $(n=4)$. Entre os periódicos editados no Brasil ( $n=4)$, publicados em língua portuguesa, observou-se que apenas um artigo teve também sua versão publicada em língua inglesa.

Cabe destacar que a despeito da temática da SI ter sido publicada em muitos títulos de periódicos $(n=16)$, o total de artigos em cada um ainda permanece baixo. Com base em informaçôes disponíveis nos sites dos periódicos, foram extraídas a descrição do escopo e das áreas de interesse das revistas listadas na Tabela 1. Os resultados apontaram que a maioria é do campo da Educação e Educaçăo Especial $(n=4)$, e, em seguida, Psicologia $(n=4)$ e Gerontologia $(n=3)$, sendo os demais das áreas de Serviço Social $(\mathrm{n}=2)$, Enfermagem $(\mathrm{n}=2)$ e Administração $(\mathrm{n}=1)$. Esses achados pressupóem que, para uma melhor compreensão da SI, é necessário um esforço interdisciplinar para o qual concorrem várias ciências e especialidades do conhecimento científico.

\subsection{Perfil dos aUtores E DAS AUTORIAS}

Verificou-se o predomínio de artigos em coautoria $(\mathrm{n}=17)$ em comparação aos individuais $(\mathrm{n}=1)$. A construção do conhecimento sobre a SI pode ser fruto de pesquisa especializada e interdisciplinar e amparada no contexto da ciência, que Ziman (1983) denomina de ciência coletivizada, em que assinala que o individualismo na pesquisa acadêmica se encontrava irreversivelmente limitado. Além disso, os artigos em coautoria denotam a colaboração científica, 
conforme assinalado por Price (1976) em seu clássico estudo sobre o desenvolvimento da ciência, que constata o crescimento das coautorias e a tendência de desaparecimento das autorias únicas. Nesse contexto, a colaboração científica promove o intercâmbio de conhecimentos e tem impacto na produtividade dos autores (Lee \& Bozeman, 2005).

Os artigos analisados $(\mathrm{n}=18)$ foram produzidos por um total de 36 autores, considerando aqueles $83,3 \%(n=30)$ que contribuíram com apenas um artigo $(n=30)$ enquanto os demais $(n=6)$ fizeram entre duas e três contribuiçóes cada um. As coautoras brasileiras (Juliana Archiza Yamashiro e Thelma Simões Matsukura) contribuíram em três artigos. Também é válido destacar a presença de apenas um autor do sexo masculino (James Emmet Gardner) com maior produtividade coautor em dois artigos.

Os achados do presente estudo sobre o gênero das autorias e coautorias dos artigos vão ao encontro do que é referido no fenômeno da segregação vertical na ciência (Schiebinger, 1999), que confina as mulheres a certos campos científicos, notadamente as Ciências Humanas e determinadas áreas da Saúde que são altamente feminizadas. Tal entendimento é corroborado pelo fato de que a formação acadêmica e/ou o campo de atuação acadêmica dos autores dos artigos são nessas áreas de conhecimento, haja vista que, do total de artigos analisados ( $\mathrm{n}=18)$, a maioria $(n=14)$ é de autores das áreas de Psicologia $(n=8)$ e de Educação $(n=6)$, seguidos por artigos $(n=4)$ de autores das áreas de Terapia Ocupacional $(n=3)$ e da Enfermagem $(n=1)$.

\subsection{Países focalizados, tipos de deficiêNCIa e POPUlaÇáo alVo dos eStudos}

$\mathrm{Na}$ identificação dos locais onde as pesquisas foram desenvolvidas, observa-se que, além de países do continente europeu (Reino Unido e Albânia), há uma diversidade de países localizados em outros continentes: Ásia (Coreia), Oriente Médio (Israel), Oceania (Austrália e Nova Zelândia), América do Norte (Canadá e Estados Unidos) e América do Sul (Brasil). Os países supracitados diferem na provisão de bem-estar, nos comportamentos demográficos e na cultura familiar, portanto essas variáveis podem interferir nos padróes de SI em famílias com crianças com deficiência. No entanto, quando esses achados são cruzados com os países de origem dos autores, os resultados são semelhantes, pois corroboram o entendimento de que os autores escolhem investigar os problemas que afetam as realidades locais.

Isso não significa, entretanto, que alguns achados dessas pesquisas não possam ser comparados aos achados de outros países e/ou regióes, explicando as diferenças na provisão de bem-estar institucional e nos sistemas de segurança social que diferem substancialmente entre os países, tanto da Europa quanto de outros continentes. (Aasve et al., 2012). Aliás, ao comentar sobre o caráter local da ciência atual, Ziman (2000) argumenta que, embora algumas soluçóes possam ser dadas "localmente", os problemas que elas respondem são muitas vezes globais.

No âmbito da SI entre pais e avós de netos com deficiência, também foi investigado quais os tipos de deficiência da população-alvo dos estudos, de acordo com a nomenclatura adotada pelos autores. 


\section{Figura 3}

Tipos de deficiência abordadas nos artigos

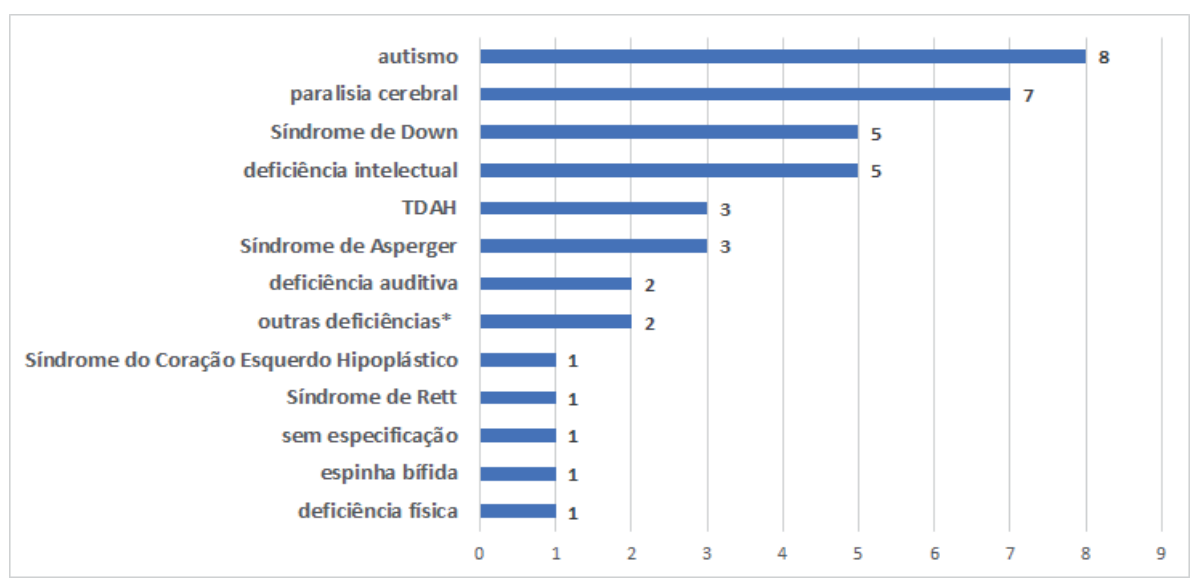

É válido observar que algumas denominações de deficiência adotadas pelos autores são diferentes das atuais, pois os artigos analisados se referem a pesquisas publicadas desde 1994.

A Figura 3 relaciona 13 tipos de deficiência da população eleita nos estudos. Entretanto, cabe destacar que o total de deficiências $(n=40)$ é superior ao total de artigos $(\mathrm{n}=18)$, pois há casos em que a amostra do estudo foi composta por pessoas com diversas deficiências e de estudos que elegeram pessoas com apenas um tipo de deficiência. Além disso, houve um artigo que não especificou o tipo de deficiência.

Notou-se que a maioria $(n=12)$ dos artigos envolveram famílias de crianças diagnosticadas com o Transtorno do Espectro Autista (TEA), a saber: Autismo ( $\mathrm{n}=8)$; Síndrome de Asperger $(n=3)$, Síndrome de Rett $(n=1)$. Em seguida, comparecem os artigos que abordaram a SI em famílias de crianças com Paralisia Cerebral $(n=7)$, Síndrome de Down $(n=5)$ e Deficiência Intelectual ( $n=5)$. Famílias com crianças com transtornos do déficit de atenção com hiperatividade (TDAH) também foram alvo dos artigos $(n=3)$. Os estudos que tiveram menor incidência foram aqueles realizados com famílias de crianças com deficiência física, auditiva e quadros patológicos, como a síndrome da hipoplasia do coração esquerdo e a espinha bífida, todos com um estudo cada.

\subsection{Dimensóes DA SOLIDARIEDADE INTERGERACIONAL (SI)}

Quase todos os estudos que utilizam a teoria de SI se concentraram em medir uma ou uma pequena seleção das dimensões do modelo (Hogerbrugge \& Komter, 2012). Na presente pesquisa, as dimensóes da SI foram identificadas a partir da leitura completa dos artigos. Apenas oito estudos abordaram apenas uma dimensão, os outros 10 estudos abordaram mais do que uma dimensão, como apresentado no Quadro 1. 


\section{Quadro 1}

Dimensóes de Solidariedade Intergeracional abordadas nos artigos

\begin{tabular}{|l|l|}
\hline Dimensóes de SI & Autores (ano) \\
\hline Associativa & $\begin{array}{l}\text { Mirfin-Veicht et al. (1997) } \\
\text { Katz \& Kessel (2002) } \\
\text { Sullivan et al. (2012) }\end{array}$ \\
\hline Funcional & $\begin{array}{l}\text { Yamashiro \& Matsukura (2014) } \\
\text { Kresak et al. (2014) }\end{array}$ \\
\hline Afetiva & Margetts et al. (2006) \\
\hline Estrutural & Mano (2016) \\
\hline Normativa & Woodbridge et al. (2011) \\
\hline Funcional e afetiva & $\begin{array}{l}\text { Gardner et al. (1994) } \\
\text { Hornby \& Ashworth (1994) } \\
\text { Matsukura \& Yamashiro (2012) } \\
\text { Woodbridge et al. (2009) }\end{array}$ \\
\hline Funcional e conflitual & Rezende et al. (2003) \\
\hline Afetiva e estrutural & Baranowski \& Schilmoeller (1999) \\
\hline Consensual e conflitual & Hastings et al. (2002) \\
\hline Conflitual e normativa & Lee \& Gardner (2015) \\
\hline Associativa, funcional e conflitual & Yamashiro \& Matsukura (2015) \\
\hline Estrutural, afetiva e funcional & Ravindran \& Rempel (2010) \\
\hline
\end{tabular}

$\mathrm{Na}$ maioria dos estudos, foram identificadas as dimensões funcional e afetiva, estando em consonância com o estudo de revisão integrativa de Leme et al. (2016), o qual envolveu 34 artigos nacionais que abordavam uma ou mais dimensóes do modelo da SIF. As dimensóes consensual e de conflito apareceram com menor frequência; dimensão consensual também foi a menos identificada por Leme et al. (2016). Observou-se que as dimensóes estão estruturalmente relacionadas entre si e a SI envolve uma construção multifacetada por meio da interdependência e das interaçóes entre as gerações, além do importante e significativo apoio dos avós aos pais e aos netos com deficiência em diferentes esferas da vida (Woodbridge et al., 2009).

\subsection{OBJETIVOS E DESFECHOS DOS ESTUDOS}

A partir da análise de conteúdo dos artigos foram identificados os principais objetivos e desfechos dos estudos. Em relação aos objetivos, verificou-se a presença daqueles que buscaram identificar e descrever experiências de avós de crianças com deficiências, conhecer seu senso de identidade, valores, papéis e componentes emocionais. Outros compararam determinadas dimensóes de SI entre avós que têm netos com deficiência versus aqueles que têm netos 
sem deficiência. O levantamento de aspectos do funcionamento familiar, como percepçóes de mães, envolvimento dos avós com irmãos de crianças com deficiências ou com outros membros da família, qualidade de vida familiar, estresse parental e o envolvimento dos avós nos cuidados diários e rotinas do cotidiano também foram objetivos abordados. Além disso, envolveram o relacionamento intergeracional, as práticas e os níveis de apoio. Em uma das pesquisas foram encontrados três temas-chave que caracterizam as visões dos avós: formação da identidade, estilos e representação de papéis.

No que se refere aos desfechos dos estudos, foi possível identificar um conjunto de cinco subtemas. É válido comentar que, em alguns estudos, esses subtemas aparecem combinados. A seguir são detalhados cada um desses subtemas.

\subsubsection{Avós COMO FONTE DE APOIO}

Grande parte dos estudos apontou, de forma direta ou indireta, os avós como importantes fontes de apoio às famílias. $\mathrm{O}$ apoio de avós pode ser modificado ao longo do tempo e traz, geralmente, benefícios para ambas as geraçóes envolvidas. Ademais a relação com os avós pode melhorar o desenvolvimento cognitivo da criança (Katz \& Kessel, 2002). Nessa mesma direção, os avós aparecem como uma garantia de bem-estar familiar em diferentes tipos de famílias, especialmente nas com crianças autistas. Avós também são uma fonte de apoio ao cuidarem de irmãos de crianças com deficiência.

Os resultados revelam também a associação de algumas variáveis com o apoio oferecido pelos avós. Os baixos níveis de apoio de avós podem estar associados à idade avançada e a problemas de saúde dos avós (Hornby \& Ashworth, 1994), sentimento de pertencimento familiar dos avós e do histórico de relacionamento familiar positivo (Mirfin-Veicht et al., 1997). Nessa perspectiva, Baranowski e Schilmoeller (1999) indicaram que a solidariedade afetiva entre as geraçóes adultas foi um preditor significativo de apoio dos avós maternos e paternos. Além disso, no estudo supracitado, a proximidade residencial foi indicada como preditor de apoio das avós, mas não dos avôs. Os resultados do estudo de Hastings et al. (2002) apontaram para a associação entre apoio dos avós e nível de estresse das mães, mas não de pais. Já Sullivan et al. (2012) destacaram que a participação das avós no processo de intervenção e o aumento da comunicação entre os membros da família podem facilitar o apoio dos avós. Por último, ainda foi identificada a existência de correlação entre fontes de apoio e qualidade de vida familiar (Kresak et al., 2014) e de associação entre o envolvimento dos avós e a satisfação no tocante ao seu papel com suas atitudes no que diz respeito às deficiências, relação com seus filhos adultos e com suas próprias experiências de vida (Katz \& Kessel, 2002).

Por outro lado, há desfechos que revelam a jornada emocional vivida pelos avós, com destaque para o processo de adaptação e aceitação da deficiência; o "duplo luto" pelo que poderia ter sido a vida tanto para o filho quanto para o neto frente à não existência da deficiência; o orgulho da habilidade familiar em ajustar-se aos desafios; e a ampliação do conceito de "dupla preocupação" para "tripla preocupação" ao direcionar o foco de pesquisa e prática para os papéis e necessidades de avós e irmãos em famílias cujos filhos menores têm doenças que ameaçam a vida (Woodbridge et al., 2009). 
Geralmente, os avós de crianças com deficiência parecem realizar práticas de apoio mais específicas e, por vezes, renunciam questóes pessoais e de cuidado com a sua saúde para ajudar as famílias dos netos (Yamashiro \& Matsukura, 2015). Mesmo com tamanha dedicação, o estudo desenvolvimento por Matsukura e Yamashiro (2012) revela o desejo de avós, bem como de irmãos de crianças com deficiência, em prestar ainda mais apoio. Por todos esses aspectos, os avós são considerados potenciais fontes de informação de aspectos gerais das crianças com deficiência e de suas famílias, foco de intervenção e de educação (Baranowski \& Schilmoeller, 1999; Margetts et al. 2006).

\subsubsection{ATUAÇÁO DE PROFISSIONAIS COM A FAMÍLIA, INCLUINDO OS AVÓS}

A maioria dos desfechos revela a importância de incluírem-se avós nos processos de intervenção da criança em diferentes modalidades e níveis de ação: grupos de intervenção, oficinas, grupos de apoio ou educativo, reunióes de diagnóstico e tratamento, visto que integram o cotidiano das famílias e se constituem como importante fonte de apoio às mães e aos netos. $\mathrm{O}$ estudo desenvolvido por Gardner et al. (1994) demonstrou que os avós, apesar de apresentarem domínio da terminologia médica, têm pouco conhecimento do processo de desenvolvimento e das intervençóes educacionais e psicológicas dos seus netos. De forma complementar, Margetts et al. (2006) reconhecem que a equipe pode melhorar, de forma indireta, a assistência oferecida às crianças ao considerar os avós como fonte de apoio à família.

É importante também encontrar estratégias alternativas para a promoção do apoio informal entre avós e filhos-adultos (Mirfin-Veicht et al., 1997). Entre as estratégias, pode-se citar a criação de condiçóes que favoreçam o aumento da comunicação entre os membros da família, visando facilitar o apoio e o envolvimento dos avós (Sullivan et al., 2012). O desenvolvimento de habilidades de comunicação pode facilitar não apenas a interação e apoio dos avós com os filhos-adultos, mas também com seus próprios netos com deficiência (Woodbridge et al., 2011). Destaca-se, ainda, a importância de oferecer espaço de escuta aos avós, visto que muitos não têm recebido apoio emocional para ajustar-se à realidade de ter um neto com deficiência (Hornby \& Ashworth, 1994). Nesse processo, não se pode negligenciar que a geração atual de avós tem, geralmente, experiência limitada com pessoas com deficiência, pois a história da deficiência ao longo de seu ciclo de vida foi marcada pela marginalização, sendo necessário, assim, o enfretamento de novos desafios e a redefinição do papel de avós (Woodbridge et al., 2011).

\subsubsection{NÍVEIS DE ESTRESSE E CONFLITOS}

Os desfechos revelam diferenças de estresse e conflitos nas famílias frente aos tipos de estudo e medidas adotadas. Há resultados que apontam não haver diferenças entre mães e pais da mesma família em termos de relatos de estresse e percepção de apoio e conflito com os avós. Outro estudo afirma que o conflito e o apoio oferecido pelos avós, conforme já citado, se associam às classificaçóes de estresse das mães, mas não dos pais (Hastings et al., 2002).

Os avós contribuem, geralmente, com papéis positivos, mas podem ser fontes de problemas aos pais em situaçóes específicas, como na participação excessiva na tomada de decisão em relação à educação dos netos; expressóes de pesar na frente destes; e percepçóes 
disfuncionais em relação à deficiência dos netos (Lee \& Gardner, 2015). De uma forma geral, os estudos apontam que o nível de estresse e de conflito não estão necessariamente ligados a ter um neto com deficiência e o tipo ou gravidade da deficiência em si. Segundo Yamashiro e Matsukura (2015), avós de crianças com deficiência e de crianças com desenvolvimento típico se sentem estressadas, mesmo aquelas que podem contar com uma rede de suporte social. Especificamente no que tange aos avós de crianças com deficiência, Woodbridge et al. (2009) destacam que eles experimentam intensa jornada emocional no momento do diagnóstico, independentemente da deficiência da criança. Assim sendo, é importante acompanhar o processo de adaptação e manejo de habilidades para superar os desafios de todos os membros da família (Woodbridge et al., 2009).

\subsubsection{DifERENÇAS E SEMELHANÇAS ENTRE AVÓS DE NETOS COM E SEM DEFICIÊNCIAS}

Apenas três estudos foram compostos por amostras mistas, formadas por avós de netos com e sem deficiências. O estudo desenvolvido por Sullivan et al. (2012), com díades mães-avós de crianças com Transtorno do Espectro Autista (TEA) e de mães-avós de crianças com desenvolvimento típico, apontou diferenças quanto ao funcionamento e ao envolvimento familiar. A presença de criança com TEA foi preditor de menores níveis de satisfação da avó, maior nível de frustação e necessidade de informação das avós. Tais condiçóes se constituem em obstáculos para o envolvimento familiar da avó. A amostra foi formada por avós que não eram cuidadores primários e vinculados a organizaçóes/serviços, não sendo possível generalizar os resultados para familiares que enfrentam maiores demandas de cuidado ou têm menos recursos de apoio.

Kresak et al. (2014) apontaram diferenças significativas entre avós que criavam netos com e sem deficiência em relação às fontes de apoio e qualidade de vida familiar. A percepção de satisfação com a qualidade de vida é maior em todos os domínios, exceto paternidade, para o subgrupo de avós de netos sem deficiências. Observaram correlação moderada entre fontes de apoio e qualidade de vida para ambos os grupos. A amostra foi composta por avós de língua inglesa que se consideravam cuidadoras primárias de, pelo menos, um neto. Assim, também deve-se ter cautela ao generalizar os resultados (Kresak et al., 2014).

Yamashiro e Matsukura (2015) observaram diferenças na presença de sintomas vivenciados de estresse entre grupos de avós de crianças sem e com deficiência. Das seis avós de crianças sem deficiência, apenas uma apresentou sintomas de estresse. Já no grupo das avós de crianças com deficiência, essa relação foi inversa. A despeito disso, os resultados qualitativos apontaram para a compreensão que ambas as avós se sentem estressadas, sendo os principais motivos: problemas de saúde, preocupação com os filhos-adultos, com os netos e com o marido. A amostra da referida pesquisa foi reduzida, formada por pessoas residentes em uma cidade do interior do estado de São Paulo. Destaca-se a necessidade de mais pesquisas para avaliar a presença de estresse e as fontes de apoio recebido pelas avós.

\subsubsection{ASPECTOS CULTURAIS E UNIVERSAIS}

Os avós, independentemente da cultura, têm tradicionalmente assumido um importante papel no cuidado de seus netos (Ravindran \& Rempel, 2010). De qualquer forma, as 
principais pesquisas enfatizam que pode haver diferenças culturais em como os avós apoiam as famílias de crianças com deficiência (Woodbridge et al., 2009).

O resultado do estudo de Woodbridge et al. (2009), composto por uma amostra de 22 avós australianos de crianças com deficiência, difere de outros estudos internacionais no que se refere ao sentimento de responsabilidade de avós em manter a família unida. Nesse caso, os avós da presente pesquisa consideram seus filhos-adultos como agente principal de proteção, cuidado pessoal e emocional aos membros da família, podendo ser definido com o papel social de "lynchpin", no inglês (Woodbridge et al., 2009).

Para Lee e Gardner (2015), as crenças e os comportamentos de avós com netos que apresentam deficiências precisam ser interpretados com base em sua cultura. Segundo os autores supracitados, a cultura asiática tradicional considera a deficiência como resultado de uma variedade de causas não desejáveis, como possessão demoníaca ou transgressóes de ancestrais e pais. Acredita-se, geralmente, que a causa da deficiência da criança é de responsabilidade da mãe e que pessoas com deficiência não podem contribuir positivamente para a família e para a sociedade, sendo frequentemente excluídas da convivência com pessoas do ambiente externo. Ademais, a responsabilidade pelo trabalho doméstico e pela educação das crianças é colocada sobre as mulheres; assim, o papel das avós nos cuidados dos netos é maior do que dos avôs. Apesar dos referidos autores destacarem que o conflito entre visóes tradicionais e contemporâneas da cultura coreana ainda ser um desafio, nota-se que outras culturas experimentam fenômenos semelhantes e passam por um processo de mudança.

Ainda no que se refere às crenças e ao comportamento, Rezende et al. (2003) focam na visão de reprodução da cultura intrafamiliar e reforçam que a identificação de conteúdo que atravessam intergeracionalmente a família pode guiar novos caminhos para lidar com a deficiência, ampliar a percepção do contexto e a necessidade da participação da família no processo de reabilitação da pessoa com deficiência auditiva, especificamente. Frente às similaridades e às diferenças encontradas, é necessário ampliar as pesquisas quantitativas e qualitativas, e as descobertas necessitam subsidiar o debate de políticas e a criação de serviços de apoio para favorecer o reconhecimento do lugar dos avós e facilitar o desempenho de seus papéis na família (Woodbridge et al., 2009).

\section{Conclusóes}

A presente pesquisa reforça a complexidade das relações familiares e a multidimensionalidade da SI, que é construída ao longo do tempo e atravessada pelo contexto social e cultural. Os avós, sobretudo as avós, permanecem como importantes fontes de apoio às famílias de crianças com e sem deficiências e podem, inclusive, influenciar no suporte oferecido por outros membros da unidade familiar.

As avós como fonte de apoio às famílias de crianças com deficiências geralmente contribuem positivamente, apesar de alguns estudos apontarem possível relação de estresse e conflito, especialmente frente à percepção heterogênea sobre a deficiência e a expressão de pesar por parte dos avós, falta de informação e histórico de relacionamento familiar negativo. O nível de estresse, de conflito e o processo de adaptação e superação de avós, mães e pais não necessariamente estão ligados ao tipo e à gravidade da deficiência e, apesar de apresentarem algumas 
particularidades, todos os membros da família experimentam intensa jornada emocional no momento do diagnóstico, sendo importante acompanhar o manejo de habilidades para superar os desafios ao longo do tempo.

Os estudos analisados envolveram diferentes dimensóes de SI, com destaque para a funcional e a afetiva. Destacam-se alguns preditores para o efetivo apoio dos avós: avós de idade não avançada e com boas condiçôes de saúde, sentimento de pertencimento familiar, histórico de relacionamento familiar positivo, proximidade residencial, participação das avós no processo de intervenção e o aumento da comunicação entre os familiares, boa qualidade de vida familiar, satisfação com o seu papel e experiências de vida. A maioria dos estudos revela a importância de incluírem-se os avós nos processos de intervenção da criança em diferentes modalidades e níveis de ação. Ademais, reforçam a necessidade de oferecer um espaço de apoio emocional a eles, de informação e de terapia voltados à melhoria da comunicação, do relacionamento familiar, do enfretamento de novos desafios e de redefinição do papel de avós.

A principal limitação da presente pesquisa envolveu o fato de estar circunscrita aos 18 estudos originais selecionados em língua inglesa e portuguesa, admitindo-se contribuiçôes nessa temática publicada por outros autores em outros idiomas e em estudos de revisão. Com relação às contribuiçôes, ressalta-se sua contemporaneidade, pois a temática é pouca estudada e pretende-se colaborar com a expansão de estudos teóricos ou empíricos nacional e internacionalmente, contribuindo não apenas com a compreensão do impacto da presença de uma criança com deficiência na família, das estratégias de apoio e dos fatores associados à solidariedade entre as geraçóes, mas também com o planejamento de intervençóes. Possibilita também avançar no estado da arte na área ao apresentar o perfil das publicaçóes, população alvo dos estudos e principais dimensóes da solidariedade intergeracional. Não se limita em apresentar as lacunas, mas as tendências para o aperfeiçoamento da produção do conhecimento sobre a temática da SI entre mães, avós e netos com deficiência. Considera-se que o estudo desenvolvido pode servir de base para estudos comparados, os quais são cada vez mais demandados no cenário da produção científica no âmbito global.

Esta pesquisa conquista destaque especial frente aos desafios enfrentados por uma sociedade que convive com o aumento progressivo da longevidade, mudanças nas estruturas familiares, transformaçóes no relacionamento e no engajamento de papéis sociais dos membros da família, mudanças nas concepções de deficiência, baixa oferta de serviços e de estratégias políticas de saúde, de educação e de assistência social pautadas na intersetorialidade que atendam a todos os integrantes da família nuclear e extensa.

\section{REFERÊNCIAS}

Aasve, A., Arpino, B., \& Goisis, A. (2012). Grandparenting and mother's labour force participation: a comparative analysis using the generations and gender survey. Demographic Research, 27(3), 53-84. https://doi.org/10.4054/DemRes.2012.27.3

Abramovay, M., Castro, M. G., Pinheiro, L. C., Lima, F. S., \& Martinelli, C. C. (2002) Juventude, violência e Vulnerabilidade Social na América Latina: desafios para políticas públicas. Brasília. UNESCO. Recuperado em 6 de julho de 2020 de https://unesdoc.unesco.org/ark:/48223/ pf0000127138 
Baranowski, M. D., \& Schilmoeller, G. L. (1999). Grandparents in the lives of grandchildren with disabilities: mother's perceptions. Education \& Treatment of Children, 22(4), 427-446.

Barbosa, M. A. M., Balieiro, M. M. F. G., \& Pettengill, M. A. M. (2012). Cuidado centrado na família no contexto da criança com deficiência e sua família: uma análise reflexiva. Texto \& Contexto: Enfermagem, 21(1), 194-199.

https://doi.org/10.1590/S0104-07072012000100022

Bardin, L. (2011). Análise de conteúdo. Edições 70.

Bengtson, V. L., \& Roberts, R. E. L. (1991). Intergenerational solidarity in aging families: an example of forma theory construction. Journal of Marriage and the Family, 53(4), 856-870. https:/doi. org/10.2307/352993

Bengtson, V. L., \& Martin, P. (2001). Families and intergenerational relationships in aging societies: comparing the United States with German-speaking countries. Zeitschrift für Gerontologie und Geriatrie, 34(3), 207-217.

Bengtson, V., Giarrusso, R., Mabry, J. B., \& Silverstein, M. (2002). Solidarity, conflict, and ambivalence: complementary or competing perspectives. Journal of Marriage and Family, 64, 568-576. https:// doi.org/10.1111/j.1741-3737.2002.00568.x

Carmo, M. E., \& Guizardi, F. L. (2018). O conceito de vulnerabilidade e seus sentidos para as políticas públicas de saúde e assistência social. Cadernos de Saúde Pública, 34(3), Artigo e00101417. https:// doi.org/10.1590/0102-311x00101417

Centro Internacional de Longevidade Brasil (2015). Envelhecimento Ativo: Um Marco Político em Resposta à Revolução da Longevidade. Recuperado em 19 de dezembro de 2019 de http://ilcbrazil.org/portugues/wp-content/uploads/sites/4/2015/12/Envelhecimento-Ativo-UmMarco-Pol\%C3\%ADtico-ILC-Brasil_web.pdf

Clarke, E. J., Preston, M., Raksin, J., \& Bengtson, V. L. (1999). Types of conflicts and tensions between older parents and adult children. The Gerontologist, 39(3), 261-270. https://doi.org/10.1093/ geront/39.3.261

Gardner, J. E., Scherman, A., Mobley, D., Brown, P., \& Schutter, M. (1994). Grandparents' beliefs regarding their role and relationship with special needs grandchildren. Education \& Treatment of Children, 17(2), 185-196

Hastings, R. P., Thomas, H., Delwiche, N. (2002). Grandparent support for families of children with Down's Syndrome. Journal of Applied Research in Intellectual Disabilities, 15(1), 97-105. https://doi. org/10.1046/j.1360-2322.2001.00097.x

Hayashi, M. C. P. I., \& Gonçalves, T. G. G. L. (2018). Estudo bibliométrico dos balanços da produção científica em Educação Especial na Revista Brasileira de Educação Especial (1999-2017). Revista Brasileira de Educação Especial, 24(Edição Especial), 135-152. http://doi.org/10.1590/s141365382418000400010

Hogerbrugge, M. J., \& Komter, A. E. (2012). Solidarity and ambivalence: comparing two perspectives on intergenerational relations using longitudinal panel data. The Journals of Psychology Series B: Psycological Sciences and Social Sciences, 67(3), 372-383. http://doi.org/10.1093/geronb/gbr157

Hornby, G., \& Ashworth, T. (1994). Grandparents' support for families who have children with disabilities. Journal of Child and Family Studies, 3(4), 403-442. 
Katz, S., \& Kessel, L. (2002). Grandparents with children with developmental disabilities: perceptions, beliefs, and involvement in their care. Issues in Comprehensive Pediatric Nursing, 25(2), 113-128. http://doi.org/10.1080/014608602900425 30

Kresak, K. E., Gallagher, P. A., \& Kelley, S. J. (2014). Grandmothers raising grandchildren with disabilities: sources of support and family quality of life. Journal of Early Intervention, 36(1), 3-17. http://doi.org/10.1177/1053815114542506

Lee, S., \& Bozeman, B. (2005). The impact of research collaboration on scientific productivity. Social Studies of Science, 35(5), 673-702. https://doi.org/10.1177/0306312705052359

Lee, M., \& Gardner, J. E. (2015). A qualitative inquiry of Korean mothers' perceptions of grandparents' roles and support for families of children with severe disabilities. International Journal of Developmental Disabilities, 61(4), 206-221. http://doi.org/10.1179/2047387714Y.0000000053

Leme, V. B. R., Falcão, A. O., Morais, G. A. de, Braz, A. C., Coimbra, S., \& Fernandes, L. de M. (2016). Solidariedade Intergeracional Familiar nas pesquisas brasileiras: revisão integrativa da literatura. Revista da SPAGESP, 17(2), 37-52.

Luvizaro, N., \& Galheigo, S. (2011). Consideraçóes sobre o cotidiano e o habitar de crianças e adolescentes em situação de acolhimento institucional em abrigo. Revista de Terapia Ocupacional da Universidade de São Paulo, 22(2), 191-199. https://doi.org/10.11606/issn.2238-6149.v22i2p191-199

Mano, E. (2016). Social support and grand parenting in autistic children families. Academic Journal of Business, Administration, Law and Social Sciences, 2(1), 193-197.

Margetts, J. K., Le Couteur, A., \& Croom, S. (2006). Families in a state of flux: the experience of grandparents in autism spectrum disorder. Child: Care, Health \& Development, 32(5), 565-575. http://doi.org/10.1111/j.1365-2214.2006.00671.x

Matsukura,T.S., \&Yamashiro, J.A. (2012). Relacionamentointergeracional, práticas deapoioecotidianode famílias de crianças com necessidades especiais. Revista Brasileira de Educação Especial, 18(4), 647-660. http://doi.org/10.1590/S1413-65382012000400008

Milanez, C. M., Córdova, Z. V. E., Castro, A., \& Fraga, C. C. (2019). O funcionamento familiar na saúde emocional e psicológica de crianças e adolescentes. Id on Line Rev. Mult. Psic. 13(47), 1-16. https://10.14295/idonline.v13i47.1905

Miranda, G. M. D., Mendes, A. C. G., \& Silva, A. L. A. (2016). O envelhecimento populacional brasileiro: desafios e consequências sociais atuais e futuras. Revista Brasileira de Geriatria e Gerontologia, 19(3), 507-519. https://doi.org/10.1590/1809-98232016019.150140.

Mirfin-Veitch, B., Bray, A., \& Watson, M. (1997). We're just that sort of family: intergenerational relationship in families including children with disabilities. Family Relations, 46(1), 305-311. https://doi.org/10.2307/585129

Price, D. S. (1976). O desenvolvimento da ciência: análise histórica, filosófica, sociológica e econômica. Livros Técnicos e Científicos.

Ravindran, V. P., \& Rempel, G. R. (2010). Grandparents and siblings of children with congenital heart disease. Journal of Advanced Nursing, 67(1), 169-175. http://doi.org/10.1111/j.13652648.2010.05482.x

Rezende, I. G., Krom, M., \& Yamada, M. O. (2003). A repetição intergeracional e o significado atual da deficiência auditiva. Psicologia: Teoria e Pesquisa, 19(2), 177-184. http://doi.org/10.1590/S010237722003000200011 
Schiebinger, L. (1999). Has feminism changed science?. Harvard University Press.

Silva, M. R., Hayashi, C. R. M., \& Hayashi, M. C. P. I. (2011). Análise bibliométrica e cientométrica: desafios para especialistas que atuam no campo. InCID: Revista de Ciência da Informação e Documentação, 2(1), 110-129. https://doi.org/10.11606/issn.2178-2075.v2i1p110-129

Sullivan, A., Winograd, G., Verkuilen, J., \& Fish, M. C. (2012). Children on autism spectrum: grandmother involvement and family functioning. Journal of Applied Research in Intellectual Disabilities, 25(5), 484-494. https://doi.org/10.1111/j.1468-3148.2012.00695.x

Woodbridge, S., Buys, L., \& Miller, E. (2009). Grandparenting a child with a disability: An emotional rollercoaster. Australasian Journal on Ageing, 28(1), 37-40. https://doi.org/10.1111/j.17416612.2008.00344.x

Woodbridge, S., Buys, L., \& Miller, E. (2011). My grandchild has a disability': impact on grandparenting identity, roles and relationships. Journal of Aging Studies, 25(4), 355-363. https://doi.org/10.1016/j. jaging.2011.01.002

Yamashiro, J. A., \& Matsukura, T. S. (2014). Apoio intergeracional em famílias com crianças com deficiência. Psicologia em Estudo, 19(4), 705-715. http://doi.org/10.1590/1413-73722419312

Yamashiro, J. A., \& Matsukura, T. S. (2015). Cotidiano e estresse de avós de crianças com deficiência e de avós de crianças com o desenvolvimento típico. Estudos Interdisciplinares sobre o Envelhecimento, 20(3), 849-863.

Ziman, J. (1983). The Bernal lecture: the collectivization of science. Proceedings of the Royal Society of London, 219(1214), 1-19. https://doi.org/10.1098/rspa.1983.0105

Ziman, J. (2000). Real science: what it is and what it means. Cambridge University Press.

Recebido em: 12/02/2020

Reformulado em: 30/07/2020

Aprovado em: 17/10/2020 
HAYASHI, M.C.P.I. et al. 